\title{
«Die Hausarztmedizin ist die ärztliche Fachdisziplin mit der höchsten Komplexität»
}

\author{
Was 2003 in einem Strassencafé in Ljubljana als Idee entstand, wird Mitte Sep- \\ tember dieses Jahres in Basel Realität: Mit der «Wonca Europe 2009 Conference» \\ findet der europäische Hausärzte-Kongress, an dem rund 4000 Hausärztinnen \\ und Hausärzte aus Europa, Übersee und der Schweiz teilnehmen werden, erst- \\ mals in der Schweiz statt. Anlass genug für ein Gespräch mit Bruno Kissling, Haus- \\ arzt in Bern und Präsident des Organisationskomitees des Basler Wonca-Kon- \\ gresses.
}

Interview: Bruno Kesseli

Zum Wonca-Kongress 2009 siehe auch das «Zu guter Letzt» von Peter Tschudi auf der letzten Seite dieser SÄZ-Ausgabe. Informationen zum Kongress und Anmeldung unter www. woncaeurope2009.org.
Wie gelang es, den Wonca-Kongress bei seiner 15. Durchführung erstmals in die Schweiz zu holen? Bruno Kissling: Dies beruht nicht auf einer Einzelaktion, sondern ist das Resultat einer kontinuierlichen Beschäftigung einer bis vor wenigen Jahren sehr kleinen und konstanten Gruppe von Schweizer Hausärztinnen und Hausärzten, die seit vielen Jahren in der Wonca oder in Wonca-Organisationen aktiv mitgewirkt und/oder regelmässig Wonca-Kongresse besucht haben. Den ernsthaften Entschluss, den Wonca-Kongress in die Schweiz zu holen, fassten Peter Tschudi* und ich zusammen mit den KollegInnen des Basler Instituts für Hausarztmedizin am Wonca-Europe-Kongress 2003 in einem Strassencafé in Ljubljana. alle 3 Jahre abgehaltenen Wonca-World-Kongresse mit den entsprechenden «Council meetings» besucht. So wurde ich mit der gesamten Organisation der Wonca und den Kongressen zunehmend vertraut.

Der Kongress steht unter dem Motto «The Fascination of Complexity - Dealing with Individuals in a Field of Uncertainty». Welche Aspekte der Hausarztmedizin sind damit besonders angesprochen?

Die Hausarztmedizin ist die ärztliche Fachdisziplin mit der höchsten Komplexität. Aus verschiedenen Gründen: Beim hausärztlichen Erstkontakt sind die gesundheitlichen Störungen oft noch wenig spezifisch ausgeprägt und sie gleichen sich oft bei einfachen und

\section{«Sogenannt «weiche Fakten» sind oft entscheidend für den therapeutischen Erfolg und die dafür nötige aktive Mitarbeit des Patienten»}

Wie wurde Ihre Idee von den Kolleginnen und Kollegen in der Schweiz aufgenommen?

Die SGAM hat das Projekt Wonca-Kongress an ihrer Jahresversammlung 2005 begeistert und einstimmig befürwortet und ideell und finanziell unterstützt. Am «Wonca Europe Council Meeting 2005» in Kos erhielten wir, ohne Kampfwahl, den Zuschlag.

Seit wann engagieren Sie sich persönlich in Sachen Wonca? Ich persönlich bin seit 2001 - im Auftrag der SGAM, die Mitglied bei Wonca ist - Schweizer Delegierter bei Wonca. Seit 2000 habe ich lückenlos alle jährlich stattfindenden Kongresse von Wonca Europe sowie die gefährlichen Leiden. Bei der Langzeitbetreuung polymorbider, chronisch kranker Menschen, steht der Hausarzt im Zentrum. Hier treten «Welten» in unterschiedlichste Wechselwirkungen mit nicht vorhersehbarem Ausgang.

\section{Was bedeutet das konkret?}

Der Patient mit seinem persönlichen Krankheitserleben (illness) und der Arzt mit seiner Diagnose des Krankheitsgeschehens (disease) müssen eine gemeinsame Sprache finden. Gemeinsam müssen Arzt und Patient den «richtigen» Weg durch die Krankheit festlegen. Dabei stehen individuelle subjektive Vorstellundes wissenschaftlichen Komitees des Wonca-Kongresses 2009. 
Freut sich auf den Wonca-Kongress in Basel:

Bruno Kissling,

Hausarzt und Präsident des Organisationskomitees, hier beim WoncaKongress 2007 in Singapur.

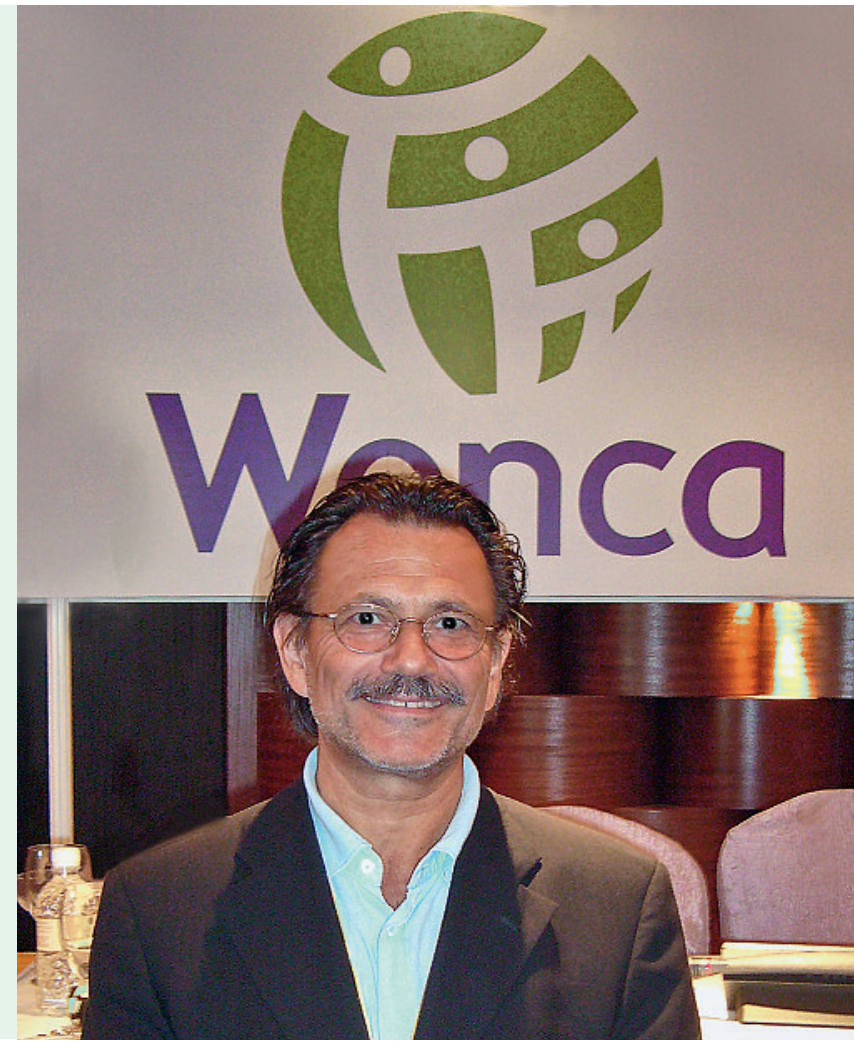

gen und Ängste des Patienten und seiner Familie im Spannungsfeld zu «objektiven» statistischen Daten. Unterschiedlichste, gleichzeitig anzuwendende Therapien interagieren. Die Koordination zwischen den verschiedenen beteiligten Spezialärzten, der Pflege, der Familie, dem Arbeitgeber, Vertrauensärzten, Mitarbeitern der IV usw. kann kaum mehr überschaubare, abenteuerliche Formen annehmen. In diesem riesigen Feld von Wechselwirkungen kann das Ganze leicht an den Rand des Chaos stossen. Bei alledem spielt die Beziehung zwischen Hausarzt und Patient eine grosse Rolle, so gross wie fachmedizinisches Know-how. Sogenannt «weiche» Fakten werden hier so bedeutsam wie «harte» Fakten und sind oft entscheidend für den therapeutischen Erfolg und die dafür nötige aktive Mitarbeit des Patienten, die «Compliance» oder «Adherence».

Wo liegen die Schwerpunkte des internationalen WoncaKongresses und des integrierten nationalen SGAM-Kongresses?

Die Schwerpunkte des wissenschaftlichen WoncaKongresses liegen weniger in spezifischen Therapieformen zu spezifischen Krankheitsbildern - «Was tut

\section{Vorstösse der SGAM bei Wonca Europe und Wonca World}

- Call for humanity, Wonca world conference in Orlando 2004, WoncaNews, Vol 31, Nr 2, April 2005, Seite 5.

Kissling B. Petition «15 by 2015» - Stärkung der Grundversorgung in den Entwicklungsländern. Schweiz Ärztezeitung. 2008;89(36):1532.
- Wonca Europe guidelines on external sponsorship, Wonca Europe conference in Paris 2007. man?». Diese wichtige fachspezifische Fortbildung wird üblicherweise an den nationalen Kongressen in den Heimatländern der Kongressteilnehmer angeboten. In Basel haben wir dafür den nationalen SGAMKongress integriert. Hier können die für den Erhalt der Dignitäten in Gynäkologie, Pädiatrie, Psychosomatik und Labormedizin nötigen Credits erworben werden.

Welches sind die «Wonca-typischen» Gebiete?

Die Wonca-Themen befassen sich mit der Lehre und Forschung in Hausarztmedizin sowie, auf einer mehr übergeordneten Ebene, mit der hausärztlichen Praxis: «Wie tut man es?» - wie praktiziert, lehrt und erforscht man eine patientenorientierte hausärztliche Gesundheitsversorgung von hoher Qualität? Im 184-stündigen Kongressprogramm finden sich Themen wie die ArztPatienten-Beziehung, ethische Fragen zur Unsicherheit und Übersicherheit, berufsphilosophischer Austausch, Reflexionen zur Förderung der Prävention und eines gesunden Lifestiles, Diskurse zum Schutz der Patienten vor dem Zuviel an Therapie und Prävention (quartenäre Prävention), Umgang mit Grenzsituationen, neue interdisziplinäre Behandlungs- und Praxisformen usw.

Gibt es neben der Fortbildungs- und wissenschaftlichen auch eine politische Komponente des Kongresses?

Wonca ist eine - unpolitische - wissenschaftliche Vereinigung. Wissenschaft und Forschung haben aber immer auch Auswirkungen mit einer politischen Dimension. Die Schweizer Hausarztmedizin steigt mit der Organisation dieses Kongresses vom hausarztmedizinischen «Schwellenland», das sie vor wenigen Jahren noch war, in die internationale Liga auf. Sie ist wissenschaftlicher und akademischer geworden - und parallel dazu politisch glaubhafter bezüglich ihrer Bedeutung in der universitären Lehre und für die Gesundheitsversorgung der Bevölkerung.

Die Hausarztmedizin ist in der Schweiz in verschiedener Hinsicht - Stichworte: Nachwuchsmangel, Tarife - unter Druck. Hat ein internationaler Hausarzt-Kongress zum jetzigen Zeitpunkt vor diesem Hintergrund eine besondere Bedeutung? Im Rahmen des Kongresses findet ja auch die Gründung des neuen Berufsverbandes «Hausärzte Schweiz» statt.

Der Wonca-Kongress kommt gerade zum richtigen Zeitpunkt. Er ist ein Meilenstein in der Geschichte der Schweizer Hausarztmedizin. Für verschiedene an-

- Swiss request for a Wonca Europe resolution on a specialist title for family medicine and a vocational training of 5 years in all European countries, Wonca Europe conference in Istanbul 2008.

- Active promotion of the petition « 15 by 2015 » and Wonca campaign, Wonca Europe conference in Istanbul 2008.* 
stehende wichtige Projekte dient er als zeitliche Zielvorgabe, so für die Gründung des neuen Berufsverbandes «Hausärzte Schweiz», die in Basel stattfinden wird. Dieser wird den Schweizer Hausärztinnen und Hausärzten einen stärkeren politischen Einfluss ermöglichen. Nicht zuletzt mit dem Wonca-Kongress vor Augen sind an vier unserer fünf Medizinischen Fakultäten Institute für Hausarztmedizin entstanden.

Gibt es weitere für die Hausarztmedizin positive Effekte? Die Forschungstätigkeit der Schweizer Hausärzte hat, eindeutig im Zusammenhang mit Wonca, stark zugenommen. Sichtbar ist dies beispielsweise an den SGAM-Kongressen mit einer wachsenden Posterausstellung zu hausärztlicher Forschung. Am Wonca-Kongress in Basel sind Schweizer Hausärzte an 178 der 1194 eingereichten Abstracts beteiligt. Sie geben, oft in internationaler Zusammenarbeit, rund 30 Workshops (von insgesamt 130), präsentieren je rund 50 Kurzvorträge (von 300) und Posters (von 555).
Präkonferenz der Jungärztinnen und Jungärzte. Sie bringen die Ideen und Bedürfnisse der künftigen Hausärzte in den Diskurs um die Zukunft der Hausarztmedizin ein, stärken die Zusammengehörigkeit der Hausärzte, gehen aktiv auf die Medizin Studierenden $z u$ - wichtige Schritte für die Attraktivität des hausärztlichen Berufs und dessen Gestaltung in der Zukunft.

Sind die Veränderungen des Berufsbildes der Hausärztin / des Hausarztes und die sich abzeichnenden neuen Grenzziehungen beispielsweise zum Arbeitsfeld der PflegeberufeStichwort: Advanced Practice Nurses - am Kongress ein Thema?

Selbstverständlich haben wir auch die Pflegeberufe zur aktiven Beteiligung an den Kongress eingeladen. Eine intensive Zusammenarbeit mit den Pflegenden ist uns wichtig. Gerade für eine gute Langzeitbetreuung der wachsenden Zahl an polymorbiden chronisch Kranken ist sie unabdingbar nötig. Dies bedeutet nicht,

\section{«Die Forschungstätigkeit der Schweizer Hausärzte hat, eindeutig im Zusammenhang mit Wonca, stark zugenommen»}

Wie ist die Ausstrahlung solcher Veranstaltungen auf den hausärztlichen Nachwuchs?

Die «Jungen Hausärztinnen und -ärzte Schweiz» sind vor zwei Jahren aus der europäischen Vereinigung der «Young Doctors» - «Vasco da Gama Movement» - hervorgegangen und haben sich kürzlich die Vereinsstruktur gegeben. In Basel organisieren sie eine europäische

\section{Was ist Wonca?}

Wonca World ist seit 1972 die Weltorganisation der Hausärzte. Wonca [1] bedeutet «World Organization of Family Doctors». Sie ist eine politisch neutrale, wissenschaftliche Vereinigung und umfasst nationale hausärztliche Fachorganisationen sowie akademische Instanzen in Lehre und Forschung von über 100 Ländern aus allen Kontinenten sowie Einzelmitglieder. Die in der Wonca organisierten Hausärzte betreuen über $80 \%$ der Weltbevölkerung! Die Ziele von Wonca sind: Verbesserung der Lebensqualität aller Menschen dieser Erde durch Förderung hoher Standards in der Hausarztmedizin; Entwicklung von akademischen Organisationen für Hausarztmedizin; Austausch unter den Mitgliedorganisationen; Repräsentation der hausärztlichen Aktivitäten in Lehre, Forschung und beruflichem Alltag. Wonca World pflegt in einigen Belangen eine enge Zusammenarbeit mit der WHO. Die SGAM ist seit 1992 Wonca-Mitglied für die Schweiz. dass sich die beiden Berufsgruppen gegenseitig ersetzen können, dass die Hausarztmedizin an Advanced Practice Nurses abgegeben werden soll. Es geht um die ständige Verbesserung der spitalexternen Patientenbetreuung durch die Gestaltung einer sich gegenseitig ergänzenden Zusammenarbeit dieser beiden Berufsgruppen.

Wonca Europe umfasst alle europäischen Länder. Ihre drei Netzwerkorganisationen EURACT (Lehre), EQuiP (Qualität) und EGPRN (Forschung) befassen sich mit allen für die Hausarztmedizin in Europa wichtigen Belangen und erarbeiten zusammen mit den Delegierten aller Mitgliedorganisationen Grundlagendokumente für die Hausarztmedizin. Diese können von den nationalen hausärztlichen Organisationen, angepasst an ihr Gesundheitssystem, weiterverwendet werden. Alle Jahre führt die Wonca Europe einen europäischen Kongress zum wissenschaftlichen Austausch durch. 2009 erstmals in der Schweiz - Basel (16.-19. September). Ein für die Schweiz sehr wichtiges Dokument ist die «European definition of general practice / family medicine» [2], revidiert 2005, an der die Schweiz mitgearbeitet hat. Sie ist Grundlage des Weiterund Fortbildungsprogramms der SGAM geworden und im Rahmen der Schweizerischen Medizinischen Interfakultätskommission SMIFK in den Lernzielkatalog der Medizinstudierenden eingeflossen. 
Der Kongress bietet reichlich Gelegenheit zum innereuropäischen Austausch. Sind in Bezug auf die Entwicklung der Hausarztmedizin in Europa Tendenzen festzustellen, aus denen sich Schlüsse für die Schweiz ziehen lassen?

Wonca ist ein Ort der Begegnung, des Austauschs und des Gegenseitig-voneinander-Lernens zur Förderung der Hausarztmedizin in allen Ländern für eine qualitativ hochstehende Gesundheitsversorgung der Bevölkerung. Es gibt in Europa eine starke Tendenz zur gezielten und praxisorientierten Weiterbildung zum Facharzt in Hausarztpraxen anstatt in Spitälern. In Holland und neu auch in Frankreich werden Assistenzärzte mit dem Berufsziel Hausarztmedizin regelmässig $\mathrm{zu}$ hausarztspezifischen Kursen zusammengezogen. Für die Grosspraxen und Notfallzentren, wie sie sich nun auch in der Schweiz entwickeln, dienen uns vor allem Dänemark und Holland als Vorbilder. Die Kon- takte zu diesen Ländern begannen in der Regel an Wonca-Kongressen. Die Vereinigung der Schweizer Jungärztinnen und -ärzte, die ich eben erwähnt habe, ist klar auf Wonca zurückzuführen.

Ist der Kongress ausschliesslich auf Hausärztinnen und Hausärzte ausgerichtet, oder ist das Zielpublikum weiter gefasst? Welche anderen Zielgruppen werden angesprochen, und mit welchen Veranstaltungen?

Das Zielpublikum sind sicher Hausärztinnen und Hausärzte, aber auch ganz besonders Assistenzärztinnen und -ärzte auf dem Weg zur Hausarztmedizin und auch Studierende mit Interesse an Hausarztmedizin. Alle anderen Spezialärztinnen und -ärzte und weitere Berufsgruppen, die sich für ein Gesundheitswesen mit einer starken Hausarztmedizin im Zentrum interessieren, sind sehr herzlich willkommen.

\section{Röntgenschutzkleidung: Bei «bleifrei» ist Vorsicht geboten}

\author{
Heinrich Eder ${ }^{a}$, \\ Waldemar Zapf \\ a Dr. Ing., vormals Bayerisches \\ Landesamt für Umwelt, \\ Augsburg \\ b MTR, Kreiskrankenhaus \\ Zwiesel
}

\section{Überblick}

Röntgenschutzkleidung wurde noch vor einigen Jahren überwiegend aus Blei oder Bleiverbindungen hergestellt. Aus Gründen der Umweltproblematik werden zunehmend bleifreie Schürzen entwickelt und in den Handel gebracht. Hierbei spielt auch der Gewichtsfaktor eine Rolle: Bleifreie Schürzen sollen vermeintlich leichter sein als Schürzen aus Blei.

Hinsichtlich der Schutzwirkung wurden bislang alle Schürzen nach dem sogenannten Bleigleichwert (PBGW) beurteilt. Sie sind in 0,25-, 0,35- und 0,50-mmBlei-Äquivalent erhältlich.

Die Frage ist nun, ob das Schutzkriterium «Bleigleichwert» auf bleifreie Schürzen bzw. Blei-Composite-Schürzen (Mischmaterial aus Blei und bleifreien Stoffen) übertragen werden kann.

Die Antwort lautet: Nein! Die Bleigleichwertkennzeichnung einer derzeit benutzten bleifreien Schürze kann deshalb in Bezug auf die tatsächliche Schutzwirkung irreführend sein [1].

Der Bleigleichwert wird nach der internationalen Norm IEC 61331-1 [2,3] im schmalen Strahlenbündel bestimmt. Diese Messmethodik erfasst lediglich die Primärstrahlung und nicht die im Material entstehende Sekundärstrahlung. Blei entwickelt im Energiebereich des diagnostischen Röntgens praktisch keine Sekundärstrahlung, deshalb ist die Methodik dort sachlich richtig. Nicht so bei bleifreien Stoffen:

Zinn, Barium und Antimon - diese Stoffe sind in Bleifrei-Schürzen häufig vertreten - werden bei äusserer Röntgen-Bestrahlung zur Fluoreszenz angeregt. Die Röntgen-Fluoreszenzstrahlung ist wie im sichtbaren Bereich (Beispiel Polarlicht) niederenergetisch, d.h. sie besitzt eine geringere Energie als die primäre Strahlung.

\section{Prudence à propos des vêtements de protection contre les radiations «sans plomb»}

Les vêtements de protection contre les radiations étaient surtout fabriqués, il y a encore quelques années, à partir de plomb ou de composés à base de plomb. On fabrique et on commercialise de plus en plus des tabliers sans plomb pour des raisons de protection de l'environnement. Le facteur poids joue ici aussi un rôle: les tabliers sans plomb seraient prétendument plus légers que ceux avec du plomb. Des mesures montrent toutefois dans beaucoup de cas, que l'exposition aux radiations est plus élevée lors du port de tablier sans plomb que celle des vêtements de protection habituelle qui en contiennent.

Les tabliers sans plomb qui n'ont pas démontré leur effet protecteur selon la norme DIN 6857-1 ne doivent plus être utilisés au cours de procédures de fluoroscopie (interventions, pose de cathéter cardiaque, angiographie, etc.). De nouveaux matériaux, en partie encore en développement, laissent espérer que dans l'avenir ils pourront offrir de facto une protection identique à celle du matériel traditionnel à base de plomb pour un poids plus faible.
Dr. Ing. H. Eder

Am Stadtpark 43

D-81243 München

eder-h@arcor.de 